\title{
Variations
}

Variations

Revue internationale de théorie critique

16 | 2012

Tahrir is here !

\section{Les relations socioprofessionnelles inter-acteurs à travers une analyse des lieux et de l'espace dans l'entreprise}

Adel Ayari

\section{(2) OpenEdition}

Journals

Édition électronique

URL : http://journals.openedition.org/variations/140

DOI : $10.4000 /$ variations. 140

ISSN : 1968-3960

Éditeur

Les amis de Variations

Édition imprimée

Date de publication : 16 janvier 2012

Référence électronique

Adel Ayari, « Les relations socioprofessionnelles inter-acteurs à travers une analyse des lieux et de l'espace dans l'entreprise », Variations [En ligne], 16 | 2012, mis en ligne le 20 février 2012, consulté le 02 mai 2019. URL : http://journals.openedition.org/variations/140 ; DOI : 10.4000/variations. 140

Ce document a été généré automatiquement le 2 mai 2019.

Les ami•e•s de Variations 


\title{
Les relations socioprofessionnelles inter- acteurs à travers une analyse des lieux et de l'espace dans l'entreprise
}

\author{
Adel Ayari
}

\section{NOTE DE L'ÉDITEUR}

Première publication sur www.theoriecritique.com, « Tahrir is here ! », 16 janvier 2012, pp. 141-149

Les deux entreprises que l'on a observées sont la S.T.A Nour et l'A.T Assad ${ }^{2}$. Elles sont les uniques fabricants industriels sur le marché local des accumulateurs électriques en plomb pour le démarrage, ou batteries de démarrage. Toutes les deux sont certifiées ISO et même pour Assad certifiée ISO-TS et ISO 14001. Toutes les deux se partagent plus de $85 \%$ du marché local et sont exportatrices. Autre point commun, elles ont une présence géographique quasi-identique qui leur permet de couvrir tout le territoire national. En effet, elles ont chacune six (6) agences dans les mêmes villes sièges de gouvernorats. Ces agences locales étaient dans chaque ville, à une époque pas très lointaine ${ }^{3}$, si proches l'une de l'autre n'étant séparées parfois que par une centaine de mètres. Une autre similitude est le fait que les deux sièges sociaux et les deux usines ${ }^{4}$ sont eux-aussi situés assez proches l'un de l'autre séparés par la RN $n^{\circ} 1$ mais éloignés seulement de quelques 300 mètres. Deux raisons principales expliquent ces points de rencontre. Primo, l'implantation des deux sites s'est faite dès le début dans deux zones industrielles presque limitrophes aménagées pour l'activité industrielle. Secundo, les deux entreprises étant très concurrentes essaient de couvrir tout le marché local et donc de bien quadriller le terrain en se positionnant de la sorte. 


\section{L'aménagement des lieux : profusion de signes et profession de sens}

2 Les similitudes sont là aussi frappantes. En effet, en se déplaçant pour visiter les deux entreprises, l'on est frappé par la multiplicité des points de ressemblance sur les deux sites. En réalité, c'est l'occupation des lieux, leur affectation, leur aménagement, le déplacement des acteurs à l'intérieur de ces espaces qui nous ont paru fort révélateurs. Pourquoi le site est-il- ainsi exploité, fait, planifié, partagé presque ? On pense qu'il existe, dans notre cas du moins, une relation étroite entre le lieu où s'est implantée l'entreprise et ce que cette dernière en fait, sa manière de l'intégrer en tant que lieu de production. Du moment de son appropriation foncière ou immobilière, le lieu n'est plus un terrain nu, il advient un élément de tout un processus de planification, d'organisation, de travail, de vente, d'activité économique, bref, d'activité sociale. En parodiant Jean-Marc Stébé, on pourrait dire qu'à l'instar de l'histoire d'une ville, il serait même évident que l'histoire de l'entreprise est inséparable de la manière dont ses lieux sont gérés ${ }^{5}$.

3 Tant la configuration architecturale des bâtiments que les signes physiques extérieurs et visuels (pancartes, signalisations, emblèmes, étendards...), indiquent une tendance claire à un confinement des lieux à la production et à l'activité économique. Cette exploitation des espaces est, en fait, partie intégrante d'une approche rationnelle et n'est pas fortuite. C'est que tout un savoir industriel et architectural est mobilisé pour mettre sur pieds les édifices et les bâtiments. Leur agencement, leur exploitation et leur mise en service sont du domaine du génie industriel, du génie civil et font partie de l'activité du bureau des méthodes au sein de l'entreprise. Donc, une des premières manifestations de la rationalité organisatrice de l'activité de l'entreprise, est ce qu'elle fait du territoire et des lieux sur lesquels est édifié le site industriel. Comment elle les exploite et les organise. En d'autres termes, l'entreprise et ses acteurs sociaux entretiennent et nouent, ce que Di Méo a désigné comme étant "des rapports novateurs avec l'espace ${ }^{6}$. Ainsi, les édifices et les bâtiments s'élevant sur ces lieux cessent, comme disait Bachelard, d'être des boîtes inertes aux simples formes géométriques. Les lieux sont porteurs d'une organisation sociale de l'espace et ce dernier «transcende l'espace géométrique ${ }^{7}$, celui de la production.

$4 \quad$ Sur les murs extérieurs des deux entreprises autant que sur ceux de l'intérieur sont collés des fiches, des pancartes en papier, des plaques en plastique et des cadres, qui renvoient tous au travail. On peut y lire et voir des directives, des photos, des textes, des consignes, qui, tous, rappellent les vertus de la discipline et l'abnégation dans le travail, font des louanges au produit fabriqué, évoquent l'altruisme commercial de l'entreprise qui pense à la satisfaction du client, exposent les prix et certificats glanés. Des murs des bureaux et du hall où sont exposés des affiches, des autocollants et même des totems, aux slogans de l'habillage des véhicules de distribution jusqu'aux motifs des stylos, des calendriers sur les murs et ceux gravés sur les cendriers sur les tables de travail ; tout évoque clairement et directement l'entreprise, ses valeurs, ses visées, son histoire et ses performances. Le lieu est investi par toute une imagerie, par des supports, où se manifestent des idées et des valeurs gestionnaires et de marketing qui ne peuvent échapper aux regards du personnel. Plus, elles encadrent, signifient, orientent, et s'imposent à travers les règles et l'idéologie qu'elles véhiculent et suscitent à tout moment de la journée, de par le quadrillage et l'endoctrinement qu'elles pratiquent. À l'une des entreprises, c'est 
l'emblème même qui est dessiné sur une bonne longueur des murs de l'enceinte extérieure. Par des dessins évoquant assez clairement l'atome, la physique, la science, la vitesse et la force on est en présence d'une fonction symbolique et même d'une icône qui ne sont pas sans rappeler l'image d'une entreprise se voulant, ou se voyant, conquérante, "sûre d'elle » et rationnelle. L'entreprise qui affirme son pouvoir et sa puissance en se dotant d'une pancarte géante visible depuis des centaines de mètres qui indique son emplacement. Un pouvoir et une puissance symbolisés par un étendard qui flotte haut, tel un drapeau national, indiquant clairement qu'ici, que là, c'est le territoire de l'entreprise, c'est son fief, son domaine. Entre pancartes, cadres, totems, emblèmes et étendards, c'est la relation forte, qui apparait, entre le lieu et ses icônes, qui deviennent « le registre des énoncés en rapport direct avec la chose qu'ils représentent $»^{8}$.

\section{L'image formée chez le personnel}

5 Les conditions de travail dans les bureaux jugées difficiles, insupportables et même inhumaines font, en outre, que "le sens du lieu reflète la qualité perçue d'un espace ", celui quotidien du travail. Les chauffeurs livreurs dans les deux entreprises, sont confrontés plus que leurs autres collègues à cette faiblesse des infrastructures administratives. Alors qu'ils sont un personnel mobile et qu'ils passent entre huit et dix heures à l'extérieur, l'absence de bureaux propres à eux s'ajoute à cette forme de « nomadisme » et l'accentue. La camionnette du chauffeur livreur se substitue au bureau et devient comme une sorte d'un point d'attache physique à l'entreprise. La mécanique est remodelée en bureau ambulant avec ses stylos, son encrier, ses trombones, ses tampons, ses bons de commandes, ses archives, ses dossiers. L'habitacle mue en une invention de dépannage administratif quotidien. Le matériel roulant s'imbibe de l'intimité de son chauffeur qui en fait aussi un lieu de restauration, de repos, de sieste entre les heures de travail. La camionnette-bureau n'est plus un moyen de livraison mais l'on y découvre une autre dimension qui «devrait résister à des métaphores qui accueillent le corps humain, l'âme humaine. Mais la transposition à l'humain se fait tout de suite dès $»^{10}$ que l'on se penche sur cette mécanique en la scrutant autrement, presque inhabituellement. C'est un bureau ambulant avec des heures de travail semblables à celles administratives. Mais destiné à un usage individuel aménagé en fonction du goût du chauffeur.

6 Même si l'on peut considérer les bureaux en tant qu'endroits et lieux de travail exprimant un aménagement industriel où tout est calculé en termes d'investissement, en coût de revient et en calcul de rentabilité ; ils ne cessent d'être un espace « qui n'est pas physique, mais un construit qui mêle concret et abstrait, matériel et immatériel, faits sociaux, faits physiques, faits d'économie $»^{11}$. Les salariés et commerciaux que l'on a rencontrés ${ }^{12}$ sont unanimes à considérer leurs bureaux et les conditions de travail globalement incompatibles avec une bonne exécution des tâches. Plus, "c'est dégradant et inhumain qu'on travaille dans de telles conditions; même les poules des usines sont mieux loties que nous $»^{13}$. De la situation des hommes au travail à celle des animaux de l'industrie alimentaire, il n'y a pas un seuil à franchir mais une image évoquant directement une autre. Plus que «le niveau d'abstraction des termes...et la cohérence d'un raisonnement ${ }^{14}$, c'est le jugement que porte les acteurs sur leur environnement immédiat qui nous intéresse. Parce qu'ils sont occupés pendant de longues années par des salariés, les bureaux, les ateliers, les comptoirs ne sont plus de simples lieux. Aucun de ces lieux et endroits ne doivent être 
considérés tel «un tableau noir sur lequel on écrit puis on efface des chiffres et des figures [...] chaque aspect, chaque détail de ce lieu a lui-même un sens $»^{15}$. Ces bureaux deviennent presque un habitat occupé à longueur de journées par des salariés qui y voient « un deuxième foyer, tellement j'y ai passé de longues années de ma vie $»^{16}$. Seulement, ce foyer n'est pas familial bien que familier. Les locaux comme tels, participent à forger ce sentiment de mal-être, cette pénibilité de travail ressentie, à rendre insupportable et pesant un quotidien qui se reproduit et qui s'éternise car pérennisé depuis des dizaines d'années.

7 En évoquant leurs lieux de travail ou pour parler de leurs entreprises, du personnel des deux entreprises, des mots et des expressions reviennent fréquemment et qui démontrent non seulement le lien affectif avec le lieu de travail mais aussi des ressentiments et des images formées. Ainsi le lieu est désigné par trou, tombeau, cimetière, prison. Ou encore il est comparé à une place où l'on meurt, où l'on étouffe, où on est enterré de son vivant. Le lieu dans ce discours n'est jamais neutre, n'est jamais un simple lieu de travail. Il est lieu d'exclusion, de charge insupportable, mais aussi de « lieu où l'on gagne son pain ». Il est évoqué comme étant une place où «l'un d'entre nous a passé la moitié de sa vie ", où "je passe plus de temps qu'auprès de ma famille ». Mais aussi associé à un passé glorieux mais perdu, à une place " où on a laissé notre sang ». Chez certains, parler du lieu de l'entreprise se fait plutôt au passé "quand les choses étaient autres ", se confondant avec « une sorte de compensation au déclin [...] et à l'effacement des groupes ouvriers qui lui étaient liés ${ }^{17}$.

Plus que des mots, plus que des paroles ou des expressions, ces qualificatifs expriment une mémoire vivante, une trame de jugements, de visions et regards formés à propos du lieu de travail et donc à propos du monde professionnel où agissent et s'activent ces acteurs. Cette mémoire est vivante parce qu'elle fonctionne à travers des rapports quotidiens avec la réalité sociale du lieu de travail, car, « l'héritage collectif est interprété par les acteurs vivants et en fonction de cette interprétation, une histoire reconstruite émerge $\aleph^{18}$. En parlant du lieu où ils travaillent, les acteurs évoquent ce qu'ils ont réalisé, ce qu'ils ont fait, leurs parcours professionnels truffés de réussites et d'échecs.

\section{Le lieu et l'espace : un autre organigramme}

En effet, au contraire des bureaux de l'écrasante majorité du personnel salarié, commercial et des autres fonctionnaires du bas de l'échelle; ceux des hauts cadres et dirigeants ainsi que ceux des actionnaires du capital, expriment d'une façon directe et clairsemée la position sociale que ces acteurs individuels occupent dans l'organigramme de l'entreprise. Ce qui leur permet de travailler dans des conditions beaucoup plus reposantes que celles des autres salariés, mais en même temps exprime une disparité révélatrice des positions sociales et du pouvoir dont jouissent certains acteurs de l'entreprise

10 L'aménagement de ces bureaux, leur ameublement, les équipements qui s'y trouvent ne peuvent être comparés à la vétusté et à l'encombrement des bureaux du personnel commercial. Les quelques pièces comptables et les copies des bons d'achat que l'on a pu consulter prouvent, sans doute aucun, l'énormité des sommes investies pour des acquisitions de toute sorte, exhibées dans des bureaux où parfois le show off orchestré et la cherté des tapis et des tables basses n'ont d'égaux que l'absence, retentissante, du goût raffiné. Encore une fois dans les bureaux se révèle au grand jour une dialectique du social 
et des lieux occupés. La distribution des moyens et des richesses se reflète et se reproduit dans la reconfiguration des lieux et crée une forte liaison et un rapport fort entre les pratiques des acteurs et les structures où ils opèrent. Les hiérarchies professionnelles dans l'entreprise sont fidèlement reproduites par un agencement entre le rang social, le pouvoir et le lieu occupé. Un agencement où sont combinés «des formes de concentration spatiale [...] des territoires circonscrits [...] des espaces appropriés [...] des espaces parcourus $»^{19}$. Les bureaux et les salles de travail sont différents à l'image des différences des rangs, des fonctions, des places et des pouvoirs des uns et des autres. Ceux de la direction générale, des directeurs et des hauts cadres, émergent comme reflétant leurs positions sociales et leurs rangs professionnels. Les autres bureaux et endroits sont ceux du reste du personnel qui sont condamnés à s'adapter à ce que l'entreprise leur a offert.

11 L'agencement des lieux permet que depuis les bureaux de la direction générale ou de ceux de la direction du personnel, il est possible de regarder ce qui se passe à la grande porte de sortie, et ainsi regarder toutes les allées et venues. Ce constat est valable pour les deux sièges des deux entreprises, ce qui ne peut être un pur hasard. Plus, le premier responsable de l'une d'entre elles a aménagé une fenêtre dans son bureau qui permet un accès visuel large et directe sur la porte principale et sur le local de la direction commerciale. Le sentiment d'être directement dans le champ visuel du PDG met mal à l'aise les occupants du bureau. L'un d'entre eux a même changé la place de sa table de travail car il estime « fort gênant, très embarrassant et pas du tout souhaitable que j'aie en face de mon regard la fenêtre du PDG $»^{20}$. C'est qu'en face, la bâtisse de la direction générale évoque et « inclut dans le visuel une arrogance [...] elle s'exhibe, se fait voir, mais pour qu'en elle chaque spectateur aperçoive l'autorité [...], la présence d'un pouvoir capable de violence ${ }^{21}$.Les endroits où sont aménagés quelques bureaux prennent une dimension disciplinaire. En effet, lorsque des cadres sont écartés de leurs fonctions qu'ils occupaient et qu'ils sont sujets à des mesures disciplinaires, la direction générale les affecte en des bureaux éloignés. Ne disposant plus du même luxe qu'avant ni du même confort, ces bureaux sont meublés juste du strict nécessaire. Il n'y a ni téléphone, ni climatisation, ni chauffage. En perdant l'ancien rang hiérarchique, son occupant perd son statut et se voit ses prérogatives annulées et perd du coup les anciennes conditions de travail. Ces bureaux sont éloignés du bâtiment de la direction générale, ils en sont à l'écart à l'image de leurs nouveaux occupants. La mise à l'écart est à double sens, celle du pouvoir et celle des lieux.

12 Entre des lieux exclusifs pour les ouvriers et d'autres exclusifs pour les agents de bureaux ; entre des espaces interdits et d'autres à l'accès réservé, on découvre un partage des lieux qui indique un partage des tâches mais qui tout de même "suppose la coordination de l'interaction dans des flots de relations situées dans l'espace et le temps et "canalisées" dans des contextes et des lieux régularisés " ${ }^{22}$.

Le mouvement et le déplacement, des acteurs itinérants et mobiles de la FDV, à l'intérieur de l'entreprise obéissent moins à un raisonnement d'efficacité technique et d'organisation qu'à celui d'une logique sociale de pouvoir et de contrôle qui fait de l'espace de l'entreprise et de ses endroits, un espace et un lieu, sinon interdits, du moins fortement contrôlés. Dès lors, le lieu et l'espace dans l'entreprise s'imprègnent de cette restriction, de ces contraintes, qui leur donnent des propriétés et des caractéristiques autres que des simples lieux de travail organisés en vue de la production. Une telle organisation de l'espace épouse les valeurs, les interdits, s'associe à des délimitations de 
la circulation du personnel de l'entreprise, pour créer et instaurer effectivement des règles d'organisation du travail. L'espace ainsi organisé crée des codes de conduite, des ordres à respecter, des signes et des pratiques, qui s'instaurent façonnent les comportements et contribuent à l'élaboration et à la mise en œuvre d'une gestion des choses et des personnes. Ce qui est primordial ici c'est « la production de l'espace, [...] l'usage de l'espace, ses propriétés qualitatives " ${ }^{23}$.

Les lieux sont divisés en des endroits strictement réservés et interdits par des pancartes, des barrières, des signalisations et parfois même des grilles. Ne sont admis à y pénétrer que ceux qui y travaillent et y ont leurs bureaux ou postes. Ce qui fait que la plupart des salariés de l'entreprise n'ont pas le droit d'aller là où bon leur semble. La plupart d'entre eux ne peuvent pénétrer dans ces lieux que pour des motifs strictement professionnels. Cependant, le constat est totalement différent pour les hauts cadres et les membres de la direction générale, qui jouissent du droit d'accéder à tous les lieux, de circuler librement à l'intérieur du site. C'est un privilège que leur procure leurs statuts. Même le parking n'est pas accessible pour tous. Sont prioritaires les hauts cadres, et parfois la direction interdit tout bonnement au personnel de l'utiliser. Elle en fait place pour le premier responsable de l'entreprise pour sa collection de voitures anciennes En fait, l'espace de l'entreprise se caractérise par cette séparation qui fait qu'il «se brise en lieux assignés (signifiés, spécialisés) et lieux interdits (à tel ou tel groupe [...]). Il se sépare en espaces $»^{24}$ correspondants moins à l'activité professionnelle des acteurs, qu'à leur position hiérarchique, sociale et à leur pouvoir. Un tel contrôle des lieux fait que ces derniers, assortis de bâtisses, de machines, de véhicules, de toute sorte d'objets anthropiques, se muent en des lieux sociaux où les différents acteurs de l'entreprise " agissent alors en fonction : de leur(s) propre(s) constat(s); des connaissances qu'ils ont du territoire ; des représentations qu'ils s'en font ; de la liberté que leur offre le système de gestion au sein duquel ils évoluent ; de leurs objectifs propres [...] Il s'agit des interrelations multiples qui lient ceux qui décident, perçoivent, s'entre-aperçoivent, s'opposent, s'allient, imposent et finalement aménagent $»^{25}$.

Tel un fait saillant, le site de l'entreprise est un espace contrôlé soit par mesure de sécurité soit par raison d'efficacité ce qui donne aux lieux mêmes une géographie du dedans et de dehors ajustée, parallèlement à la stratification sociale des acteurs. L'organisation du travail s'identifie en partie à sa planification des lieux et l'exploitation qu'elle en fait, et «bien qu'elle obéisse à des nécessités techniques aussi rigoureuses que les lois de la nature, [elle] constitue bien une création artificielle et se conforme aux règles d'une logique intellectuelle et collective $\aleph^{26}$, celle rationnelle, dirigeante et efficace.

Ainsi est mis en place un système de gestion des lieux où tout ce qui se passe est contrôlé, épié et rapporté. De l'usine aux entrepôts, du local administratif aux postes des gardiens, entre les murs extérieurs et ceux de l'intérieur, l'entreprise, telle une institution disciplinaire, « explicitement s'apparente au couvent, à la forteresse, à une ville close [... ]À chaque individu sa place, et en chaque emplacement, un individu [...] l'espace disciplinaire tend à se diviser en autant de parcelles qu'il y a de corps ou d'éléments à répartir. Il faut annuler les effets des répartitions indécises, la disparition incontrôlée des individus, leur circulation diffuse, leur coagulation inutilisable et dangereuse ; tactique d'anti désertion, d'anti vagabondage, d'anti agglomération. Il s'agit d'établir les présences et les absences, de savoir où et comment retrouver les individus[...] mesurer les qualités ou les mérites [...] Il s'agit à la fois de distribuer les individus dans un espace où on peut les isoler et les repérer; mais aussi d'articuler cette distribution sur un appareil de 
production qui a ses exigences propres [...] Dans la discipline, [...] chacun se définit par la place qu'il occupe dans une série, et par l'écart qui le sépare des autres. L'unité n'y est donc ni le territoire (unité de domination), ni le lieu (unité de résidence), mais le rang : la place qu'on occupe dans un classement $»^{27}$.

\section{Conclusion}

17 L'occupation des espaces et des lieux, dans ces deux entreprises, reflète un certain mode de management et de gouvernance. Son analyse nous a permis de découvrir une des facettes des relations socioprofessionnelles inter-acteurs. Son analyse contribue à la saisie de ce qu'il advient dans le monde social de l'entreprise.

\section{NOTES}

1. Cette observation s'est effectuée courant 2010 lors des multiples déplacements faits par l'auteur pour les besoins de sa thèse de doctorat soutenue à Tunis.

2. Respectivement: la société tunisienne de l'accumulateur NOUR et l'accumulateur tunisien ASSAD.

3. Depuis seulement quelques années et suite au déménagement de deux agences Nour et d'une agence d'Assad, les locaux régionaux de ces deux entreprises dans trois villes (Sousse, Gabès et Jendouba) sont éloignés de quelques kilomètres.

4. L'usine d'Assad pour les batteries de démarrage est implantée depuis presque 10 ans dans un autre gouvernorat, celui de Nabeul. Toutefois elle a aménagé l'ancienne usine pour la production des batteries industrielles.

5. STEBE J-M. avec la collaboration de MATHIEU-FRITZ A. (2001), Architecture, urbanistique et société. Idéologies et représentations dans le monde urbain, Paris, L'Harmattan, p. 132.

6. DI MEO G. (1988), Les démocraties industrielles. Crise et mutation de l'espace. Paris, Masson, p. 34.

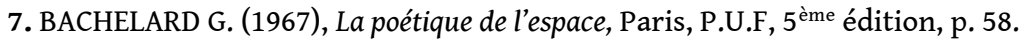

8. LUSSAULT M. (2003), «Emblème national ", in, Dictionnaire de la géographie et de l'espace des sociétés, op.cit, p. 305.

9. BAILLY A., POCOCK D. (1995) «L'humanisme en géographie », in BAILLY A. et al., Les concepts de la géographie humaine, Paris, Masson, p. 168.

10. BACHELARD G., op.cit., p. 59

11. MATTHEY L. (2005), "Le quotidien des systèmes territoriaux », in Articulo, revue de sciences humaines [en ligne], mis en ligne le 24/10/2005, consulté le 19/12/2009. URL: http:// articulo.revues.org/index $903 . \mathrm{html}$

12. On a rencontré en tout quarante-deux personnes appartenant aux deux entreprises.

13. Samir N., agent commercial

14. GRISE J-B., VERGES P., SILEM A. (1987), Salariés face aux nouvelles technologies : vers une approche sociologique des représentations sociales. Editions du CNRS, centre régional de publications de Lyon, p. 21

15. HALBWACHS M. (1968), La mémoire collective, Paris, PUF, p. 133

16. Salwa A., agent commercial 
17. DAUMAS J-C. (2006), "L'usine, la mémoire et l'histoire », in La mémoire de l'industrie, de l'usine au patrimoine, DAUMAS J-C. (dir), Presses universitaires de Franche-Comté, p. 19

18. CHENEVEZ A. (2006), "La Saline d'Arc-et-Senans, mémoire perdue de l'industrie », in, La mémoire de l'industrie, de l'usine au patrimoine, op.cit, p. 157

19. ROULLEAU-BERGER L., "Villes en friches : précarités, socialisations, compétences », in Futur antérieur $n^{\circ} 29: 1995 / 3$. Mise en ligne septembre 1995. http://multitudes.samizdat.net

20. Nader N., agent commercial

21. LEFEBVRE H. (2000), La production de l'espace, Paris, éditions Anthropos, 4 ème édition, p. 117

22. GIDDENS A. (1987), La constitution de la société, Paris, PUF, 1 ère édition, p. 127

23. LEFEBVRE H., op.cit, p. 465

24. Ibid, op.cit, p. 368

25. MOINE A. (2006), «Le territoire comme un système complexe : un concept opératoire pour l'aménagement et la géographie ", in, Espace géographique n²-2006, p. 115-132

26. HALBWACHS M. (1972), Classes sociales et morphologie, les éditions de Minuit, p. 65

27. FOUCAULT M. (1975), Surveiller et punir. Naissance de la prison, Paris, éditions Gallimard, pp. 167-171

\section{RÉSUMÉS}

À partir d'une observation sur le terrain ${ }^{1}$, on démontrera que la gestion et l'aménagement des sites (usine, ateliers, locaux administratifs et sièges sociaux) de deux entreprises industrielles tunisiennes sont moins une réponse à des contraintes technico-productives qu'une illustration d'un type de rapports socioprofessionnels inter-acteurs.

\section{AUTEUR}

\section{ADEL AYARI}

Docteur et maître-assistant en sociologie à l'Université de Tunis. Il a participé à une recherche internationale sur les transformations du marché de l'emploi tunisien en 2009 sous la responsabilité d'Alexander Neumann. 